\title{
Haemosporidioses in wild Eurasian blackbirds (Turdus merula) and song thrushes (T. philomelos): an in situ hybridization study with emphasis on exo- erythrocytic parasite burden
}

\section{Tanja Himmel}

Veterinarmedizinische Universitat Wien

Josef Harl

Veterinarmedizinische Universitat Wien

\section{Simone Pfanner}

Veterinarmedizinische Universitat Wien

Nora Nedorost

Veterinarmedizinische Universitat Wien

Norbert Nowotny

Veterinarmedizinische Universitat Wien

Herbert Weissenböck ( $\nabla$ herbert.weissenboeck@vetmeduni.ac.at)

University of Veterinary Medicine Vienna https://orcid.org/0000-0001-8197-6379

\section{Research}

Keywords: Plasmodium, Haemoproteus, Leucocytozoon, exo-erythrocytic stages, megalomeront, chromogenic in situ hybridization, virulence, Plasmodium matutinum, Plasmodium vaughani

Posted Date: December 17th, 2019

DOI: https://doi.org/10.21203/rs.2.19034/v1

License: (c) (i) This work is licensed under a Creative Commons Attribution 4.0 International License. Read Full License

Version of Record: A version of this preprint was published at Malaria Journal on February 12th, 2020. See the published version at https://doi.org/10.1186/s12936-020-3147-6. 


\section{Abstract}

Background Passerine birds are frequently infected with diverse haemosporidian parasites. While infections are traditionally considered benign in wild birds, recent studies demonstrated mortalities of passerine species due to exo-erythrocytic development of the parasites, which can damage organs in affected hosts. However, exo-erythrocytic development remains insufficiently investigated for most haemosporidian species and thus little is known about the virulence of tissue stages in wild passerine birds. The aim of the present study was to investigate natural haemosporidian infections in deceased Eurasian blackbirds (Turdus merula) and song thrushes (T. philomelos) and to determine parasite burden and associated histological effects.

Methods For molecular analysis, blood and tissue samples from 306 thrushes were screened for Plasmodium, Haemoproteus and Leucocytozoon parasites by nested PCR. For the detection of parasite stages in organ samples, tissue sections were subjected to chromogenic in situ hybridization using genus- and species-specific probes targeting the rRNAs of parasites. Exo-erythrocytic parasite load was semi-quantitatively assessed and histological lesions were evaluated in haematoxylin-eosin-stained sections.

Results 179 of 277 Eurasian blackbirds and 15 of 29 song thrushes were positive for haemosporidians. Parasites of all three genera were detected, with Plasmodium matutinum LINN1 and P. vaughani SYAT05 showing the highest prevalences. $\mathrm{CISH}$ revealed significant differences in exo-erythrocytic parasite burden between lineages in Eurasian blackbirds, with P. matutinum LINN1 frequently causing high parasite loads in various organs that were associated with histological alterations. Song thrushes infected with P. matutinum LINN1 and birds infected with other haemosporidian lineages showed mostly low parasite burdens. Two Eurasian blackbirds infected with Leucocytozoon sp. TUMER01 showed megalomeronts in various organs that were associated with inflammatory reactions and necroses.

Conclusion This study suggests that P. matutinum LINN1, a common lineage among native thrushes, regularly causes high exo-erythrocytic parasite burdens in Eurasian blackbirds, which may result in disease and mortalities, indicating its high pathogenic potential. The findings further illustrate that the same parasite lineage may show different levels of virulence in related bird species which should be considered when assessing the pathogenicity of haemosporidian parasite species. Finally, the study

provides evidence of virulent Leucocytozoon sp. TUMER01 infections in two Eurasian blackbirds caused by megalomeront formation.

\section{Background}

Avian haemosporidians compose a highly diverse group of apicomplexan parasites that occur almost globally and infect a wide range of different bird species from most families [1]. The three major genera Plasmodium, Haemoproteus, and Leucocytozoon comprise more than 200 morphologically described 
species [1] and almost 4000 unique mitochondrial cytochrome b (cytb) lineages have been recorded to date [2].

Wild passerine birds (Passeriformes) are commonly infected with haemosporidian parasites belonging to all three genera [3] and more than one third of all valid haemosporidian species have been found in this bird order $[1,4]$. The parasites harboured by passerine birds vary greatly in prevalence and their level of host-specificity, ranging from generalists, which infect a wide range of passerine species, to extreme hostspecialists which confine to single species [5-8]. Haemosporidian infections in passerine birds are usually considered benign, as individuals are often chronically infected exhibiting low levels of parasitaemia and rarely showing signs of illness $[1,9]$. The observed low virulence is explained by evolutionary reciprocal adaption of birds and parasites over long time. In contrast, infection of immunologically naïve host species regularly results in severe morbidity and has caused mortalities in several non-passerine bird species [10-18]. However, several experimental infection studies demonstrated also high susceptibility of passerine bird species to certain haemosporidian parasites and markedly different pathogenicity depending both on infected host species and parasite strain [19-23]. Moreover, few reports showed lethal infections with Plasmodium spp. in wild passerine birds which commonly harbour haemosporidian parasites [24-26].

During infection, the parasites undergo complex development in the bird host, which includes several generations of asexual replication in tissue cells (exo-erythrocytic merogony) and invasion of blood cells with gametocyte formation resulting in parasitaemia, which causes haemolysis and manifests in anaemia. Notably, in the above-mentioned cases, pathology was largely induced by severe burdens of exo-erythrocytic stages (meronts) in endothelial cells and other tissue cells in various organs. The detailed pattern of exo-erythrocytic merogony may vary substantially across both parasite and infected host species and remains unknown for the majority of haemosporidian species, particularly from the genera Haemoproteus and Leucocytozoon [27], because these life stages are difficult to access, especially in wild birds. Together with host-related factors, which presumably influence parasite development, this renders general predictions about their pathogenic impact and virulence in diverse bird hosts difficult.

Traditionally, exo-erythrocytic parasite stages are examined by histological techniques, but their detection is cumbersome because the stages are usually small and easily confounded with cellular structures or debris. In order to facilitate the detection in tissue sections and identification of parasites belonging to the three major genera, molecular probes have been developed $[14,28]$.

The present study intended to investigate avian haemosporidian infections in a large collection of Eurasian blackbirds (Turdus merula) and song thrushes (Turdus philomelos) which were found dead in Vienna and suburban regions. This investigation extends earlier research, which showed an association of Plasmodium spp. infections with mortalities of several passerine species native to Austria [25] and aims to determine the burden of Plasmodium, Haemoproteus and Leucocytozoon parasites in tissue 
samples of deceased birds and to evaluate their histological impact by chromogenic in situ hybridization, a combined molecular-histological approach.

\section{Material And Methods}

\section{Samples}

Blood and tissue samples of 306 thrushes (Turdidae, Passeriformes) including 277 blackbirds (Turdus merula) and 29 Song thrushes (T. philomelos) were obtained from the Institute of Pathology at the University of Veterinary Medicine Vienna (Austria). The samples were collected during post-mortem examinations of dead birds submitted between 2002 and 2018. Most birds were found between July and December in different parts of Vienna and surrounding districts of the federal state of Lower Austria, few birds originated from the neighbouring countries Switzerland and Hungary. A small number of the samples was already analysed earlier by Dinhopl et al. [25], however, they were screened using the primers Palu-F and Palu-R by Martínez et al. [29], which do not allow detection of Leucocytozoon parasites. In order to re-evaluate these samples using a current standard PCR assay [30] and recently developed molecular probes [28], they were included in this study as well. Tissue samples were taken from several organs, including heart, lung, liver, spleen, kidney, brain, gizzard and intestine in most cases and in some also skeletal muscle and bursa of Fabricius. For histology and chromogenic in situ hybridization (ClSH) of tissue sections, samples were fixed in formalin and embedded in paraffin wax (FFPE). For molecular analysis, tissue samples were frozen and stored at $-80^{\circ} \mathrm{C}$ until further use.

\section{Molecular analysis}

DNA was extracted from organ homogenates [31] and from dried blood spots using the DNeasy Blood \& Tissue Kit (Qiagen, Venlo, Netherlands) following the manufacturer's recommendations with the exception that two elution steps with each $100 \mu \mathrm{AE}$ buffer were performed, the second of which was used as template for PCR. For detection of haemosporidians, a nested PCR protocol [30] targeting a section of the mitochondrial cytochrome b gene $(c y t b)$ of parasites was used. First, the primers HaemNFI and HaemNR3 were used to amplify DNA of Plasmodium, Haemoproteus and Leucocytozoon. For the second PCR, the primer pairs HaemF/HaemR2 and HaemFL/HaemR2L were used to amplify DNA of Plasmodium/ Haemoproteus, and Leucocytozoon, respectively.

PCR was performed in $25 \mu \mathrm{l}$ reaction volumes containing $14.375 \mu$ nuclease-free water, $5 \mu \mathrm{l}$ X Green GoTaq Flexi Buffer (Promega, Madison, Wisconsin, USA), $2 \mu \mathrm{MgCl}_{2}$ solution (25 mM), $0.5 \mu \mathrm{l} \mathrm{PCR}$ nucleotide mix (10 mM, Promega), $0.125 \mu \mathrm{l} \mathrm{GoTaq} \mathrm{G2} \mathrm{Flexi} \mathrm{DNA} \mathrm{Polymerase} \mathrm{(} 5 \mathrm{u} / \mu \mathrm{l}$, Promega), each $1 \mu \mathrm{l}$ forward and reverse primer $(10 \mathrm{pmol} / \mu \mathrm{l})$, and $1 \mu \mathrm{l}$ of DNA template. For the second PCRs, instead of a DNA template, $1 \mu \mathrm{l}$ amplicon from the first PCR was used. Thermocycler conditions included initial denaturation at $94^{\circ} \mathrm{C}$ for $2 \mathrm{~min}, 35 \mathrm{cycles}$ of denaturation at $94^{\circ} \mathrm{C}$ for $30 \mathrm{sec}$, primer annealing at $50{ }^{\circ} \mathrm{C}$ for $30 \mathrm{sec}$ and extension at $72{ }^{\circ} \mathrm{C}$ for $1 \mathrm{~min}$, followed by a final extension at $72{ }^{\circ} \mathrm{C}$ for $10 \mathrm{~min}$. PCR 
products were visualized on 1\% agarose gels with Midori Green Advance (Nippon Genetics Europe, Dueren, Germany) using a BioSens SC-Series 710 gel documentation system (GenXpress, Wiener Neudorf, Austria). In every PCR run, positive controls (tissue samples confirmed positive in earlier screenings) and

negative controls (nuclease free water instead of DNA templates) were included. PCR amplicons from the second PCRs were sent to Microsynth Austria for bi-directional sequencing. Obtained sequences were edited and aligned in Bioedit [32] and electropherograms were carefully checked for ambiguities. In case sequences contained ambiguous characters indicating double infections, electropherograms were carefully re-checked and sequences were un-phased using DnaSP v.6.12.3 [33]. Sequences were subjected to BLAST search using the avian malaria database MalAvi [2] and NCBI GenBank. Haplotypes not showing $100 \%$ matches with published cytb lineages were given new lineage names according to MalAvi rules.

\section{Chromogenic in situ hybridization}

For the detection of parasites in tissue sections, FFPE tissue blocks of each individual bird were cut into 1-2 $\mu \mathrm{m}$ thick sections, one of which was stained with haematoxylin and eosin (HE) and the remaining ones were subjected to $\mathrm{CISH}$. Chromogenic in situ hybridization was performed following previously established protocols using genus-specific oligonucleotide probes for the detection of Plasmodium, Haemoproteus, and Leucocytozoon [14,28]. In cases of mixed infections, as determined by PCR and sequencing, several sections were separately incubated with all relevant probes. For a detailed analysis of double infections with Plasmodium vaughani and Plasmodium matutinum, species-specific oligonucleotide probes were designed based on $18 \mathrm{~S}$ ribosomal RNA gene sequences published by Harl et al. [34]. These probes were tested on tissue sections from birds infected with the respective parasites (single infections) and cross-reactions were ruled out by negative results. Tissue sections from birds harbouring those two lineages were then separately incubated with the species-specific probes. Sequences of all probes used in this study are listed in Table 1.

\section{Approximate position of Table 1.}

For $\mathrm{ClSH}$, tissue sections were deparaffinized in Neo-Clear solution (Merck Millipore, Burlington, Massachusetts, USA), rehydrated in a series of graded ethanol $(100 \%, 96 \%, 70 \%)$ and distilled water and pre-treated with proteinase $\mathrm{K}$ (Roche, Basel, Switzerland) $3 \mu \mathrm{g} / \mathrm{ml}$ in $0.5 \mathrm{M}$ Tris-HCl buffered saline at 37 ${ }^{\circ} \mathrm{C}$ for $40 \mathrm{~min}$. After proteolysis, sections were rinsed in distilled water, rehydrated in $96 \%$ and $100 \%$ ethanol and air-dried. For hybridization, tissue sections were incubated with hybridization solution containing $11 \mu$ ldistilled water, $20 \mu \mathrm{l} 20 \mathrm{X}$ saline-sodium citrate (SSC) buffer, $50 \mu$ l formamide, $5 \mu$ l herring sperm, $2 \mu \mathrm{l} 50 \mathrm{X}$ Denhardt's solution, $10 \mu \mathrm{l}$ dextran sulphate $(50 \%, \mathrm{w} / \mathrm{v})$ and $1 \mathrm{ng}$ probe per $100 \mu \mathrm{l}$ 
overnight in a humid chamber at $40^{\circ} \mathrm{C}$. On the next day, sections were subjected to stringency washes in 2X, $1 \mathrm{X}$ and $0.1 \mathrm{X}$ SSC buffer 10 min each and pre-incubated with Tris- $\mathrm{HCl}$ buffered saline containing normal goat serum and $10 \%$ Triton X-100 for 30 min before application of anti-digoxigenin-AP Fabfragments (Roche) at a concentration of 1:200 for $1 \mathrm{~h}$ at room temperature. After washing in distilled water $2 \times 15$ min, chromogenic detection was accomplished by application of NBT/BCIP (nitro-blue tetrazolium chloride/5-bromo-4-chloro-3'-indolyphosphate $\mathrm{p}$-toluidine salt, Roche) mixed with levamisole in 0.1 M Tris-buffered saline ( $\mathrm{pH} 9.5)$ and incubation for a minimum of $40 \mathrm{~min}$ in a dark, humid chamber. Enzymatic reaction was stopped by treating slides with Tris-EDTA buffer (pH 8.0) for 10 min. Finally, sections were counterstained with haematoxylin and mounted using Aquatex (Merck Millipore) and coverslips.

\section{Microscopy \& evaluation}

$\mathrm{CISH}$-treated tissue sections were analysed by bright field microscopy using 100x, 200x, 400x and 1000x magnifications. All organ samples from each bird were examined and the degree of parasite load was assessed using a semi-quantitative scoring system. Hereby, a clear differentiation was made between $\mathrm{CISH}$ signals deriving from infected blood cells, corresponding to erythrocytic stages of parasites, and signals matching exo-erythrocytic stages (tissue meronts). The extent of exo-erythrocytic merogony was assessed for each organ sample by a 0 to 3 score system $(0=$ negative, no meronts detected; $1=$ low number of meronts - not in every 200x field; 2 = moderate number of meronts - one or more meronts in several high-power fields (HPF); 3 = high number of meronts - one or more meronts in every HPF).

Microphotographs were acquired using an Olympus BX51 microscope (Olympus Europa, Hamburg, Germany) equipped with an Olympus DP71 camera. Images were adjusted for brightness and contrast and assembled in Adobe Photoshop CC 2019 (Adobe, San José, California, USA).

\section{Statistics}

Analyses were performed using the statistical software package IBM SPSS Statistics Version 24.0 (IBM Corp., Armonk, N.Y., USA). To evaluate for an association between infection intensity and parasite lineage, a Fisher's exact test was used and calculated for samples sizes $>20$. Level of significance was set at 0.05 with $* p<0.05,{ }^{\star *} p<0.01$ and ${ }^{* \star *} p<0.001$.

\section{Results}

\section{Molecular analysis}

By PCR, 194 (63.4\%) of 306 birds were positive for Plasmodium, Haemoproteus, Leucocytozoon or a combination of these genera (Table 2). Plasmodium showed the highest prevalence with 177 infected 
birds, Haemoproteus and Leucocytozoon were less prevalent with only 15 and 17 positive birds, respectively.

Of 194 positive birds, 40 (20.6\%) showed mixed infections, including 35 double and 5 triple infections. The mixed infection rate was highest among Leucocytozoon-positive birds, with 15 (88.2\%) of 17 showing a co-infection with either Plasmodium (9 cases), Haemoproteus (5 cases) or multiple Leucocytozoon lineages (1 case). Thirty-four (19.2\%) of 177 Plasmodium-infected birds harboured multiple Plasmodium lineages (24 cases) or were co-infected with Leucocytozoon (9 cases) or Haemoproteus (1 case). Six (40.0\%) of 15 Haemoproteus-infected birds showed mixed infections with Leucocytozoon (5 cases) or Plasmodium (1 case).

By sequencing, 26 unique cytb lineages (Plasmodium: 12, Leucocytozoon: 10, and Haemoproteus: 4) were identified, 13 of which were $100 \%$ identical to previously reported lineages in MalAvi or Genbank (Table 2).

\section{Approximate position of Table 2.}

The by far most prevalent lineages were LINN1, a lineage of $P$. matutinum, recorded in $99(51.0 \%)$ of 194 infected birds, and SYAT05, a lineage of $P$. vaughani, detected in 89 birds (45.9\%). Eight birds featured lineages showing 99\% matches with SYAT05 (TUMER13, TUMER14, TUMER15, TUMER16) and LINN1 (TUPHI08, TUPHI09, TUPH10). Among Leucocytozoon, TUMER01 was most prevalent, detected in seven Eurasian blackbirds. From the genus Haemoproteus, the three lineages TUPHI01, TURDUS2 and TUCHR01, all linked to the morphospecies Haemoproteus minutus, were identified. One blackbird harboured a sequence (TUMER17) matching $99 \%$ with TURDUS2.

\section{Chromogenic in situ hybridization}

All 194 birds (179 Eurasian blackbirds and 15 song thrushes) positive for haemosporidian parasites by PCR and sequencing were analyzed by $\mathrm{CISH}$, including 154 single and 40 mixed infections. $\mathrm{CISH}$ revealed parasites in tissue sections of 178 cases $(91.8 \%)$ whereas $16(8.2 \%)$ remained negative. The detected parasite stages were classified as blood or tissue stages based on the shape, size and location of signals. Blood stages typically showed oval to roundish signals not larger than blood cells and were located in capillaries and larger vessels. Signals exceeding the size of blood cells and showing variable shapes were considered exo-erythrocytic meronts (Additional file 1). In unclear cases, e.g., when the signals were all similar and in the size of blood cells, the corresponding HE-stained sections were checked for exo-erythrocytic meronts. 


\section{Detection of Plasmodium}

CISH detected Plasmodium parasites in 165 (93.2\%) of 177 PCR-positive birds, but not all birds exhibited exo-erythrocytic stages of the parasites in the tissues. $113(68.5 \%)$ cases showed both erythrocytic and exo-erythrocytic stages of parasites, whereas $46(27.9 \%)$ cases only showed exo-erythrocytic but no blood stages. Conversely, six cases (3.6\%) presented blood stages but no exo-erythrocytic meronts.

Parasite load, in terms of exo-erythrocytic merogony, varied extensively among individuals, ranging from zero to high numbers of detectable meronts. Representative photographs of low-, moderate- and highgrade parasite loads are shown for the brain and the spleen (Additional file 2). Of 159 cases that presented exo-erythrocytic stages, 74 (46.6\%) exhibited low numbers of meronts in at least one organ, 34 $(21.4 \%)$ had moderate numbers of meronts in the tissues and 51 (32.1\%) showed high loads of exoerythrocytic meronts (Table 3). Comparing Eurasian blackbirds and song thrushes, the overall percentage of exo-erythrocytic-positive cases was similar with $89.8 \%$ (149/166) and 90.9\% (10/11), respectively. However, in contrast to blackbirds, which showed various degrees of parasite merogony in their tissues, most song thrushes only exhibited low numbers of exo-erythrocytic meronts.

\section{Approximate position of Table 3.}

The distribution of exo-erythrocytic stages in different tissues ranged among individuals from single affected organs to a generalized occurrence. Regardless of the parasite lineage, certain organs exhibited exo-erythrocytic stages more frequently than others (Figure 1). The spleen was most commonly affected with a rate of $86.7 \%(144 / 166)$, followed by lung $(65.0 \%, 93 / 143)$ and brain $(61.0 \%, 100 / 164)$ (Figure 1$)$. In about $50 \%$ of the cases, tissue meronts were detected in the heart (87/168), liver (80/152), gizzard $(68 / 129)$ and bursa of Fabricius $(13 / 25)$, whereas kidney $(40.4 \%, 55 / 136)$, intestine $(34.7 \%, 33 / 95)$ and skeletal muscle $(18.2 \%, 2 / 11)$ were less commonly affected.

\section{Approximate position of Figure 1.}

Evaluation of histological lesions associated with the presence of meronts was difficult because tissues of the submitted dead birds showed varying degrees of autolysis and freezing artefacts. Histological alterations such as necrosis and inflammatory cell infiltration were most often observed in spleen, liver, lung, bursa of Fabricius and intestine and were in some cases associated with excessive exo-erythrocytic merogony of the parasites (Figure 2a). Apart from 'regular' tissue stages, some peculiar observations 
were made during histological examination of tissues. In a few $P$. matutinum and $P$. vaughani-infected birds, clusters of several exo-erythrocytic meronts were noticed in the brain. Some of the meronts had an untypical appearance as they seemed to divide into multiple smaller parts of similar size, which contained numerous merozoites (Figure 2b). Another frequent observation was the accumulation of erythrocytic parasite stages in the microvasculature of fat tissue and the serosal layers of visceral organs, most frequently in the heart and the gastrointestinal tract (Figure $2 \mathrm{c}$ and $2 \mathrm{~d}$ ).

\section{Approximate position of Figure 2.}

To evaluate whether infection intensity depended on parasite lineage, birds with single infections of the two most prevalent lineages $P$. matutinum $(n=65)$ and $P$. vaughani $(n=62)$ were compared. Because of the low number of song thrushes in the data set and to rule out host species as a confounding factor, only blackbirds were included in this analysis. The P. matutinum-infected group showed higher percentages of moderate and high-grade infections in all organs compared to the $P$. vaughani-infected group with a significant difference found for the heart, lung, kidney, brain and gizzard, but not the liver, spleen and intestine (Fisher's exact, heart $(n=121): p=0.016$; lung $(n=102): p=0.026$; kidney $(n=97): p$ $=0.043$; brain $(n=117): p<0.001$; gizzard $(n=92): p<0.001$; liver $(n=109): p=0.896$; spleen $(n=120)$ : $p=0.30$; intestine $(n=64): p=0.066)$ (Figure 3). In $P$. vaughani-infected birds high-grade parasite load was noticed only in the heart, lung, liver and spleen, whereas in $P$. matutinum-infected birds high-grade parasite load was observed in almost all organs, with brain $(25 \%, 16 / 64)$, lung $(22 \%, 12 / 59)$ and heart $(18 \%, 11 / 67)$ being most frequently affected.

\section{Approximate position of Figure 3.}

According to the PCR and sequencing results, nineteen birds (all Eurasian blackbirds) harboured coinfections with both $P$. matutinum LINN1 and $P$. vaughani SYAT05. Using the general Plasmodium probe, $\mathrm{CISH}$ detected exo-erythrocytic stages in 17 of these birds, showing moderate to high parasite loads in twelve cases and low parasite loads in five birds. Application of probes specific for either $P$. matutinum or $P$. vaughani to discern their relative roles in co-infections yielded ambiguous $\mathrm{CISH}$-results. Meronts of $P$. matutinum were detected in all 17 cases, whereas $\mathrm{CISH}$ using the $P$. vaughani-specific probe resulted in only eight positive cases, seven of which exhibited tissue stages. In three of the seven cases that yielded positive signals with both probes, meronts of $P$. vaughani and $P$. matutinum were equally abundant with few differences regarding tissue distribution, whereas in four cases meronts of $P$. vaughani were more abundant in most organs. Interestingly, in almost half of the cases, the overall abundance of meronts 
detected by both species-specific probes was lower compared to the general Plasmodium probe, indicating lower sensitivity of the species-specific probes.

\section{Detection of Haemoproteus}

In nine of 15 Haemoproteus-infected birds, parasites were detected by $\mathrm{CISH}$. In all cases, signals were few in number and restricted to larger vessels and capillaries of different organs, corresponding to blood stages of the parasites, indicating low parasitaemia. In none of the cases, exo-erythrocytic meronts were observed.

\section{Detection of Leucocytozoon}

CISH revealed parasite stages in all Leucocytozoon-infections with exception of birds infected with Leucocytozoon sp. ASOT06 (three birds). In most cases, however, only blood stages were detected. Two of seven blackbirds that were infected with Leucocytozoon sp. TUMER01 showed large tissue stages (megalomeronts) in various organs (Figure 4). In one bird, megalomeronts were detected in the heart, bursa of Fabricius and kidney. They appeared as densely packed homogenous masses of merozoites which were enclosed by thin capsules and surrounded by cells of unclear origin (Figure 4a). In the other bird, several megalomeronts were detected in the kidney, which appeared either intact or disintegrated. In the HE-stained sections, inflammatory reactions and necroses were associated with megalomeronts.

\section{Approximate position of Figure 4.}

\section{Discussion}

This study investigated natural haemosporidian infections in deceased thrushes from Austria, building on the observations of an earlier study which demonstrated an association of passerine bird mortalities with infections of Plasmodium spp. [25]. By using a combined molecular genetic and histological approach, the aim was to detect parasites of the genera Plasmodium, Haemoproteus, and Leucocytozoon in Eurasian blackbirds and song thrushes and to determine the burden of exo-erythrocytic stages in their tissues and associated histological effects.

The study showed that Eurasian blackbirds and song thrushes were frequently infected with haemosporidian parasites, particularly with Plasmodium spp., among which the lineages P. matutinum LINN1 (belonging to the subgenus Haemamoeba), and P. vaughani SYAT05 (subgenus Novyella) were predominant. High Plasmodium infection rates have been reported in these host species not only from Austria [25], but also from other localities in Europe [24,35-38] and in thrushes introduced to New 
Zealand [39], suggesting that these birds are natural reservoir hosts for Plasmodium parasites. In contrast to the study by Dinhopl et al. [25], which detected only single infections of Plasmodium spp., the present investigation further showed low prevalences of Haemoproteus spp. and Leucocytozoon spp. and a mixed infection rate of $20.6 \%$. The use of different PCR protocols and a larger sample size in this study probably explain the contrasting results. While the primers used in the study by Dinhopl et al. target only Plasmodium and Haemoproteus parasites, the primers and a nested PCR protocol used here allow detection of all three haemosporidian genera.

This investigation demonstrates that Eurasian blackbirds were regularly (up to $30 \%$ ) affected by severe burdens of exo-erythrocytic stages of Plasmodium spp. in their organs. As several cases showed, high loads of tissue meronts were associated with histologic lesions, indicating that severe multiplication of the parasites causes deleterious effects on tissues. These findings are in accordance with the observations by Dinhopl et al. in several native passerine bird species [25], emphasizing the pathogenic potential of Plasmodium parasites in natural hosts. Besides confirming earlier findings, the present study further revealed that the degree of parasite burden was associated with parasite lineage, as shown by the comparison of Eurasian blackbirds infected with either one of the two most prevalent lineages, P. matutinum LINN1 and P. vaughani SYAT05. In contrast to P. vaughani-infected birds, in which extensive tissue merogony was rarely detected, P. matutinum-infected birds were frequently affected by severe burdens of exo-erythrocytic stages, suggesting differences in virulence of these two lineages. Different strains of P. matutinum, which belongs to the subgenus Haemamoeba, have been reported to show variable levels of virulence in avian hosts [40], and previous studies already indicated that infection with LINN1 is associated with disease and mortalities [24-26]. In the present study numerous phanerozoites of P. matutinum LINN1 were particularly often detected in heart, lung, and brain of birds with high parasite burdens, similar to a previous report of fatal Plasmodium infection caused by this lineage [26]. It seems likely that the severely affected birds in this study also died due to infection with LINN1, which points out the high pathogenic potential of this lineage in Eurasian blackbirds.

Interestingly, song thrushes infected with P. matutinum LINN1 mostly showed low parasite burden. Only one of eight birds exhibited high amounts of exo-erythrocytic parasite stages in some of the organs. This song thrush harboured a mixed infection with P. matutinum LINN1 and Plasmodium sp. TUPHI08, a lineage almost identical to LINN1. It appears, that single infections with P. matutinum LINN1 in song thrushes are not as virulent as in Eurasian blackbirds, but parasite load might increase in doubleinfections. However, this remains speculative, as song thrushes were underrepresented preventing direct comparison of parasite burdens between the two host species.

There is limited knowledge on the pathogenicity of P. vaughani SYAT05, but generally, the virulence of members of the subgenus Novyella, to which P. vaughani belongs, is considered to be low [41]. The findings of the present study provide evidence that infection with P. vaughani SYAT05 can result in high parasite burdens in Eurasian blackbirds, however, the low numbers of severely affected birds suggest that this occurs infrequently. 
It is important to note, that the infected birds in this study all died naturally, and it remains open, to what extent they suffered from concomitant diseases or other pathogens that might have favoured the severity of malaria infections. Birds of the family Turdidae, in particular Eurasian blackbirds, are highly susceptible to Usutu virus (USUV) infection, and epizootic USUV-associated bird mortalities have been reported from several European countries, including Austria [31, 42-50]. More recent studies reported high USUV and Plasmodium co-infection rates in Eurasian blackbirds, suggesting that co-infection increases the likeliness of mortalities in infected birds [24, 42]. In contrast to these reports, in the current investigation, USUV and Plasmodium infections were weakly associated. A large number of birds used in this study was collected during an earlier USUV surveillance program and tested for USUV, however, of 161 Plasmodium-positive thrushes, only 35 blackbirds showed concurrent USUV infection. More importantly, most USUV-positive birds exhibited very low levels of parasite stages both in the blood and in tissues and birds showing high parasite loads were all USUV-negative, ruling out USUV co-infection as a major driver of Plasmodium parasite burden.

The results showed that co-infections with parasites of the two lineages P. matutinum LINN1 and P. vaughani SYAT05 were frequent among mixed infections. The presence of multiple parasite lineages in the same host can result in within-host competition leading to increased virulence [51, 52], but the modes of parasite interactions in avian haemosporidian co-infections remain largely unknown. Experimental studies demonstrated that concurrent infection with Plasmodium spp. can alter infection dynamics of involved lineages and result in synergistic effects on parasitaemia levels in certain hosts [53]. In the present study, species-specific probes were applied to assess the relative burden of $P$. matutinum and $P$. vaughani in the co-infected Eurasian blackbirds, which yielded ambiguous results. While tissue meronts of $P$. matutinum were seen in all cases, the corresponding parasite stages of $P$. vaughani were detected in only half of the cases, suggesting low P. vaughani abundance in these birds. In the double-positive cases, tissue stages of both parasite species were equally abundant, or tissue stages of P. vaughani were predominant. Unfortunately, from these results, it is impossible to draw any meaningful conclusions on parasite interactions in these co-infections because of two reasons. First, the apparent lower sensitivity of the species-specific probes compared to the genus-specific probes probably obscured true parasite loads. The reason for the inconsistent results and the weak robustness of the species-specific probes is difficult to explain, but might relate to poor accessibility of selected rRNA target regions due to the complex higher-order structure of these molecules [54]. The efficiency of hybridization of the probes might be improved by simply targeting a different rRNA region. However, this has to be tested in future studies. Second, and more importantly, any conclusions from the observed patterns are limited by the lack of historical information, e.g., time or order of infection, which might have been completely different in the examined individuals. Ideally, to study the effects of parasite-interaction on the development of involved parasite species during co-infections experimental approaches should be considered. In this context, sensitive species-specific probes could support the study of exo-erythrocytic development of parasites during co-infections in future studies.

As noted in the results section, some remarkable observations were made concerning the appearance and location of parasite stages in several birds infected with P. matutinum and P. vaughani. Although it was 
not the scope of this study to further pursue detailed investigation of these findings, they might be relevant in regard to the developmental biology of Plasmodium in avian hosts and are worth some comments. First, some exo-erythrocytic meronts seemed to comprise multiple smaller merozoites containing compartments. These substructures strongly resemble cytomeres, portions of developing merozoites that are formed in meronts of Haemoproteus and Leucocytozoon parasites. However, cytomeres have not been reported in Plasmodium parasites so far [27] and detailed examinations are required to rule out that the compartments are the result of co-infection of single host cells with multiple merozoites, each producing a separate meront. Second, erythrocytic parasite stages were found to accumulate in the microvasculature of fat tissue and serosal layers of visceral organs. Sequestration, i.e., adhesion of infected red blood cells to the endothelium of capillaries in certain tissues, including brain, lung, intestine and fat tissue, is a common phenomenon of human and rodent malaria parasites and has been shown to contribute to disease severity $[55,56]$. It is plausible, that avian Plasmodium parasites share the ability to sequester in the microvasculature, however, this is unexplored in birds. It is worth mentioning that the accumulation of erythrocytic parasite stages became apparent due to the strong signals in $\mathrm{CISH}$-treated sections and would have been missed in HE-stainings. Likewise, because it is difficult to detect infected blood cells in HE-stained tissue sections, the phenomenon of avian Plasmodium to retain in certain tissues might have been overlooked in the past.

In contrast to Plasmodium spp., parasite burden by Haemoproteus and Leucocytozoon spp. was low in most birds. Blood stages of Haemoproteus and Leucocytozoon detected in blood cells of infected thrushes indicate that the parasites completed their development within the host and birds died during chronic infection. Only in birds infected with Leucocytozoon sp. ASOT06, parasite stages were not observed. This Leucocytozoon lineage has been identified in species of Strigiformes from Europe [34, 57] and is probably host-specific, which explains why the parasites could not establish in the thrushes and suggests that these were accidental infections.

Two blackbirds infected with Leucocytozoon sp. TUMER01 exhibited disseminated megalomeront formation in several organs. This lineage has been previously reported in Eurasian blackbirds and clusters with other Leucocytozoon lineages found in European hosts $[38,58]$, but has not been linked to a morphologically described species yet. The megalomeronts looked quite different and appeared to be of different developmental maturity. Importantly, an enlarged host cell nucleus (central body) characteristic for Leucocytozoon megalomeronts and which distinguishes them from megalomeronts of the genus Haemoproteus [27], was not evident in all stages. This demonstrates that identification of parasites solely based on morphological characters is not always feasible and stresses the importance of parallel use of molecular detection methods such as in situ hybridization. The histology of the two blackbirds showed that the megalomeronts were associated with tissue damage and inflammatory reactions in different organs. Mortality of these birds could not be definitely linked to Leucocytozoon, because other factors than concurrent USUV-infections that might have attributed to death, were not ruled out. However, the histological findings suggest that these birds suffered from leucocytozoonosis. Notably, blood stages of TUMER01 were found in all infected birds, indicating that the parasites underwent primary merogony in all of these cases. However, except of the two birds showing megalomeronts, exo-erythrocytic stages 
were not observed. It might be the case that meronts were scarce in these birds and thus missed by examination of tissue sections, but the results rather suggest that megalomeronts were not formed in these birds. Available data from previous studies indicate that the exo-erythrocytic development of Leucocytozoon parasites is flexible and the occurrence of certain stages of the same parasite species can be variable in different host species [27]. The findings from this study suggest that the exoerythrocytic development might show some degree of flexibility even within the same host species. Leucocytozoon sp. TUMER01 obviously does form megalomeronts in Eurasian blackbirds, but the driving forces of megalomeront formation and whether these stages always appear during regular development remain unclear. To understand the role of megalomeronts in the life cycle of Leucocytozoon parasites and their pathogenicity in common hosts, further research is needed.

\section{Conclusion}

The present study provides insight into haemosporidian parasite burden in naturally infected Eurasian blackbirds and song thrushes native to Austria. The findings suggest that P. matutinum LINN1, a common lineage among thrushes, regularly causes high exo-erythrocytic parasite burdens in Eurasian blackbirds, which may result in disease and mortalities pointing out its pathogenic potential. The low parasite burdens in song thrushes infected with LINN1 illustrate that the same parasite lineage can show different levels of virulence in related bird species, which should be considered when assessing the pathogenicity of haemosporidian parasite species. Finally, the study provides evidence of virulent Leucocytozoon sp. TUMER01 infections in two Eurasian blackbirds due to disseminated megalomeront formation. Because little is known about the role of megalomeronts in Leucocytozoon in general, more research on the exo-erythrocytic development is needed to understand the pathogenicity of these stages.

\section{Abbreviations}

cytb:cytochrome b; CISH:chromogenic in situ hybridization; FFPE:formalin-fixed paraffin-embedded; HE:haematoxylin-eosin; Tris-HCl:Tris-hydrochloride; SSC:saline-sodium citrate; NBT:nitro-blue tetrazolium chloride; BCIP:5-bromo-4-chloro-3'-indolyphosphate p-toluidine salt; HPF:high-power field

\section{Declarations}

\section{Ethics approval and consent to participate}

The study was part of the FWF-project P 29628 and approved by the institutional ethics and animal welfare committee and the national authority according to $\S \S 26 \mathrm{ff}$. of Animal Experiments Acts, Tierversuchsgesetz 2012 - TVG 2012, Austria (BMWFW-68.205/0036-WF/V/3b/2017). 
Not applicable.

\section{Availability of data and material}

The dataset supporting the conclusions of this article is included within the article and its additional files.

\section{Competing interests}

The authors declare that they have no competing interests.

\section{Funding}

The study was funded by the FWF Austrian Science Fund (grant P 29628).

\section{Authors' contributions}

TH, HW and JH: study conception and design; $\mathrm{TH}, \mathrm{JH}, \mathrm{NN}$ : molecular laboratory work and analyses; JH: probe design, sequence analyses; $\mathrm{TH}, \mathrm{SP}, \mathrm{NN}$ : procedure and analyses of $\mathrm{CISH}$ experiments; $\mathrm{TH}, \mathrm{HW}$ : histopathological analysis; TH: statistical analysis and drafting of manuscript; all authors read, revised and approved the manuscript.

\section{Acknowledgements}

We thank Christiane Weissenbacher-Lang from the Institute of Pathology, Vetmeduni Vienna, and Alexander Tichy from the Platform Bioinformatics and Biostatistics for their support with statistical analyses. Alexandra Scope and Cornelia Konicek from the Clinical Unit of Internal Medicine Small Animals, Vetmeduni Vienna, are acknowledged for their cooperation and for providing blood samples from birds. We would also like to thank Gediminas Valkiūnas from the P.B. Šivickis Laboratory of Parasitology, Nature Research Centre Vilnius, for his help and comments on the histomorphology of tissue stages. Finally, we thank the FWF Austrian Science Fund for funding this work.

\section{References}

1. Valkiūnas G. Avian malaria parasites and other haemosporidia. 1st ed. Boca Raton: CRC Press; 2005. 
2. Bensch S, Hellgren O, Pérez-Tris J. MalAvi: a public database of malaria parasites and related haemosporidians in avian hosts based on mitochondrial cytochrome b lineages. Mol Ecol Resour. 2009;9:1353-8.

3. Scheuerlein A, Ricklefs RE. Prevalence of blood parasites in European passeriform birds. Proc Biol Sci. 2004;271:1363-70.

4. Braga EM, Silveira P, Belo NO, Valkiūnas G. Recent advances in the study of avian malaria: an overview with an emphasis on the distribution of Plasmodium spp in Brazil. Mem Inst Oswaldo Cruz. 2011;106 Suppl:3-11.

5. Bensch S, Stjernman M, Hasselquist D, Ostman O, Hansson B, Westerdahl H, et al. Host specificity in avian blood parasites: a study of Plasmodium and Haemoproteus mitochondrial DNA amplified from birds. Proc Biol Sci. 2000;267:1583-9.

6. Ventim R, Tenreiro P, Grade N, Encarnação P, Araújo M, Mendes L, et al. Characterization of haemosporidian infections in warblers and sparrows at south-western European reed beds. $\mathrm{J}$ Ornithol. 2012;153:505-12.

7. Križanauskienė A, Hellgren O, Kosarev V, Sokolov L, Bensch S, Valkiūnas G. Variation in host specificity between species of avian hemosporidian parasites: evidence from parasite morphology and cytochrome B gene sequences. J Parasitol. 2006;92:1319-24.

8. Van Hemert C, Meixell BW, Smith MM, Handel CM. Prevalence and diversity of avian blood parasites in a resident northern passerine. Parasit Vectors. 2019;12:292.

9. Atkinson CT, Dusek RJ, Lease JK. Serological responses and immunity to superinfection with avian malaria in experimentally-infected Hawaii amakihi. J Wildl Dis. 2001;37:20-7.

10. Donovan TA, Schrenzel M, Tucker TA, Pessier AP, Stalis IH. Hepatic hemorrhage, hemocoelom, and sudden death due to Haemoproteus infection in passerine birds: eleven cases. J Vet Diagn Invest. 2008;20:304-13.

11. Hill AG, Howe L, Gartrell BD, Alley MR. Prevalence of Leucocytozoon spp, in the endangered yelloweyed penguin Megadyptes antipodes. Parasitology. 2010;137:1477-85.

12. Taunde PA, Bianchi MV, Perles L, da Silva FS, Guim TN, Stadler RA, et al. Pathological and molecular characterization of avian malaria in captive Magellanic penguins (Spheniscus magellanicus) in South America. Parasitol Res. 2019;118:599-606.

13. Vanstreels RET, Dutra DDA, Ferreira-Junior FC, Hurtado R, Egert L, Mayorga LFSP, et al. Epidemiology, hematology, and unusual morphological characteristics of Plasmodium during an avian malaria outbreak in penguins in Brazil. Parasitol Res. 2019;118:3497-508.

14. Dinhopl N, Mostegl MM, Richter B, Nedorost N, Maderner A, Fragner K, et al. Application of in-situ hybridization for the detection and identification of avian malaria parasites in paraffin waxembedded tissues from captive penguins. Avian Pathol. 2011;40:315-20.

15. Olias P, Wegelin M, Zenker W, Freter S, Gruber AD, Klopfleisch R. Avian malaria deaths in parrots, Europe. Emerg Infect Dis. 2011;17:950-2. 
16. Valkiūnas G, Pendl H, Olias P. New Haemoproteus parasite of parrots, with remarks on the virulence of haemoproteids in naive avian hosts. Acta Trop. 2017;176:256-62.

17. Ortiz-Catedral L, Brunton D, Stidworthy MF, Elsheikha HM, Pennycott T, Schulze C, et al. Haemoproteus minutus is highly virulent for Australasian and South American parrots. Parasit Vectors. 2019;12:40.

18. Jia T, Huang X, Valkiūnas G, Yang M, Zheng C, Pu T, et al. Malaria parasites and related haemosporidians cause mortality in cranes: a study on the parasites diversity, prevalence and distribution in Beijing Zoo. Malar J. 2018;17:234.

19. Ilgūnas M, Bukauskaitè D, Palinauskas V, lezhova TA, Dinhopl N, Nedorost N, et al. Mortality and pathology in birds due to Plasmodium (Giovannolaia) homocircumflexum infection, with emphasis on the exoerythrocytic development of avian malaria parasites. Malar J. 2016;15:256.

20. Dimitrov D, Palinauskas V, lezhova TA, Bernotienè R, llgūnas $M$, Bukauskaite $D$, et al. Plasmodium spp.: an experimental study on vertebrate host susceptibility to avian malaria. Exp Parasitol. 2015;148:1-16.

21. Ilgūnas $M$, Palinauskas $V$, Platonova E, lezhova $T$, Valkiūnas $G$. The experimental study on susceptibility of common European songbirds to Plasmodium elongatum (lineage pGRW6), a widespread avian malaria parasite. Malar J. 2019;18:290.

22. Ilgūnas M, Bukauskaitė D, Palinauskas V, lezhova T, Fragner K, Platonova E, et al. Patterns of Plasmodium homocircumflexum virulence in experimentally infected passerine birds. Malar $\mathrm{J}$. 2019;18:174.

23. Palinauskas V, Valkiūnas G, Bolshakov C V., Bensch S. Plasmodium relictum (lineage P-SGS1): effects on experimentally infected passerine birds. Exp Parasitol. 2008;120:372-80.

24. Rouffaer LO, Steensels M, Verlinden M, Vervaeke M, Boonyarittichaikij R, Martel A, et al. Usutu virus epizootic and Plasmodium coinfection in Eurasian blackbirds ( Turdus merula) in Flanders, Belgium. J Wildl Dis. 2018;54:859-62.

25. Dinhopl N, Nedorost N, Mostegl MM, Weissenbacher-Lang C, Weissenböck H. In situ hybridization and sequence analysis reveal an association of Plasmodium spp. with mortalities in wild passerine birds in Austria. Parasitol Res. 2015;114:1455-62.

26. Howe L, Castro IC, Schoener ER, Hunter S, Barraclough RK, Alley MR. Malaria parasites (Plasmodium spp.) infecting introduced, native and endemic New Zealand birds. Parasitol Res. 2012;110:913-23.

27. Valkiūnas G, lezhova TA. Exo-erythrocytic development of avian malaria and related haemosporidian parasites. Malar J. 2017;16:101.

28. Himmel T, Harl J, Kübber-Heiss A, Konicek C, Fernández N, Juan-Sallés C, et al. Molecular probes for the identification of avian Haemoproteus and Leucocytozoon parasites in tissue sections by chromogenic in situ hybridization. Parasit Vectors. 2019;12:282.

29. Martínez J, Martínez-DE LA Puente J, Herrero J, Del Cerro S, Lobato E, Rivero-DE Aguilar J, et al. A restriction site to differentiate Plasmodium and Haemoproteus infections in birds: on the inefficiency of general primers for detection of mixed infections. Parasitology. 2009;136:713-22. 
30. Hellgren O, Waldenström J, Bensch S. A new PCR assay for simultaneous studies of Leucocytozoon, Plasmodium, and Haemoproteus from avian blood. J Parasitol. 2004;90:797-802.

31. Weissenböck H, Kolodziejek J, Url A, Lussy H, Rebel-Bauder B, Nowotny N. Emergence of Usutu virus, an African mosquito-borne flavivirus of the Japanese encephalitis virus group, central Europe. Emerg Infect Dis. 2002;8:652-6.

32. Hall TA. BioEdit: a user-friendly biological sequences alignment editor and analysis program for Windows 95/98/NT. Nucleic Acids Symp Ser. 1999;41:95-8.

33. Rozas J, Ferrer-Mata A, Sánchez-DelBarrio JC, Guirao-Rico S, Librado P, Ramos-Onsins SE, et al. DnaSP 6: DNA Sequence Polymorphism Analysis of Large Data Sets. Mol Biol Evol. 2017;34:3299302.

34. Harl J, Himmel T, Valkiūnas G, Weissenböck H. The nuclear $18 \mathrm{~S}$ ribosomal DNAs of avian haemosporidian parasites. Malar J. 2019;18:305.

35. Hatchwell BJ, Wood MJ, Anwar M, Perrins CM. The prevalence and ecology of the haematozoan parasites of European blackbirds, Turdus merula. Can J Zool. 2000;78:684-7.

36. Bentz S, Rigaud T, Barroca M, Martin-Laurent F, Bru D, Moreau J, et al. Sensitive measure of prevalence and parasitaemia of haemosporidia from European blackbird (Turdus merula) populations: value of PCR-RFLP and quantitative PCR. Parasitology. 2006;133:685-92.

37. Carbó-Ramírez P, Zuria I, Schaefer HM, Santiago-Alarcon D. Avian haemosporidians at three environmentally contrasting urban greenspaces. J Urban Ecol. 2017;3:1-11.

38. Hellgren $\mathrm{O}$, Križanauskienė A, Hasselquist D, Bensch S. Low haemosporidian diversity and one keyhost species in a bird malaria community on a mid-Atlantic island (São Miguel, Azores). J Wildl Dis. 2011;47:849-59.

39. Schoener ER, Tompkins DM, Parker KA, Howe L, Castro I. Presence and diversity of mixed avian Plasmodium spp. infections in introduced birds whose distribution overlapped with threatened New Zealand endemic birds. N Z Vet J. 2019;0:1-6.

40. Valkiūnas G, Ilgūnas $M$, Bukauskaitè D, Palinauskas V, Bernotienè R, lezhova TA. Molecular characterization and distribution of Plasmodium matutinum, a common avian malaria parasite. Parasitology. 2017;1-10.

41. lezhova TA, Valkiünas G, Bairlein F. Vertebrate host specificity of two avian malaria parasites of the subgenus Novyella: Plasmodium nucleophilum and Plasmodium vaughani. J Parasitol. 2005;91:472-4.

42. Rijks JM, Kik ML, Slaterus R, Foppen R, Stroo A, IJzer J, et al. Widespread Usutu virus outbreak in birds in the Netherlands, 2016. Eurosurveill. 2016;21.

43. Bakonyi T, Erdélyi K, Brunthaler R, Dán Á, Weissenböck H, Nowotny N. Usutu virus, Austria and Hungary, 2010-2016. Emerg Microbes Infect. 2017;6:e85.

44. Manarolla G, Bakonyi T, Gallazzi D, Crosta L, Weissenböck H, Dorrestein GM, et al. Usutu virus in wild birds in northern Italy. Vet Microbiol. 2010;141:159-63. 
45. Höfle U, Gamino V, de Mera IGF, Mangold AJ, Ortíz J-A, de la Fuente J. Usutu virus in migratory song thrushes, Spain. Emerg Infect Dis. 2013;19:1173-5.

46. Hubálek Z, Rudolf I, Čapek M, Bakonyi T, Betášová L, Nowotny N. Usutu virus in blackbirds (Turdus merula), Czech Republic, 2011-2012. Transbound Emerg Dis. 2014;61:273-6.

47. Chvala S, Kolodziejek J, Nowotny N, Weissenböck H. Pathology and viral distribution in fatal Usutu virus infections of birds from the 2001 and 2002 outbreaks in Austria. J Comp Pathol. 2004;131:176-85.

48. Meister T, Lussy H, Bakonyi T, Sikutová S, Rudolf I, Vogl W, et al. Serological evidence of continuing high Usutu virus (Flaviviridae) activity and establishment of herd immunity in wild birds in Austria. Vet Microbiol. 2008;127:237-48.

49. Cadar D, Lühken R, van der Jeugd H, Garigliany M, Ziegler U, Keller M, et al. Widespread activity of multiple lineages of Usutu virus, western Europe, 2016. Eurosurveill. 2017;22:1-7.

50. Weidinger P, Kolodziejek J, Bakonyi T, Brunthaler R, Erdélyi K, Weissenböck H, et al. Different dynamics of Usutu virus infections in Austria and Hungary, 2017-2018. Transbound Emerg Dis. 2019;1-10.

51. de Roode JC, Pansini R, Cheesman SJ, Helinski MEH, Huijben S, Wargo AR, et al. Virulence and competitive ability in genetically diverse malaria infections. Proc Natl Acad Sci U S A. 2005;102:7624-8.

52. Bell AS, de Roode JC, Sim D, Read AF. Within-host competition in genetically diverse malaria infections: parasite virulence and competitive success. Evolution. 2006;60:1358-71.

53. Palinauskas V, Valkiūnas G, Bolshakov C V., Bensch S. Plasmodium relictum (lineage SGS1) and Plasmodium ashfordi (lineage GRW2): the effects of the co-infection on experimentally infected passerine birds. Exp Parasitol. 2011;127:527-33.

54. Behrens S, Fuchs BM, Mueller F, Amann R. Is the in situ accessibility of the 16S rRNA of Escherichia coli for Cy3-labeled oligonucleotide probes predicted by a three-dimensional structure model of the 30S ribosomal subunit? Appl Environ Microbiol. 2003;69:4935-41.

55. Franke-Fayard B, Fonager J, Braks A, Khan SM, Janse CJ. Sequestration and tissue accumulation of human malaria parasites: can we learn anything from rodent models of malaria? PLoS Pathog. 2010;6:e1001032.

56. Cowman AF, Healer J, Marapana D, Marsh K. Malaria: Biology and disease. Cell. 2016;167:610-24.

57. Ishak HD, Dumbacher JP, Anderson NL, Keane JJ, Valkiūnas G, Haig SM, et al. Blood parasites in owls with conservation implications for the Spotted Owl (Strix occidentalis). PLoS One. 2008;3:e2304.

58. Padilla DP, Illera JC, Gonzalez-Quevedo C, Villalba M, Richardson DS. Factors affecting the distribution of haemosporidian parasites within an oceanic island. Int J Parasitol. 2017;47:225-35.

\section{Tables}


Table 1 Oligonucleotide probes used for the detection of haemosporidian parasites by chromogenic in situ hybridization

\begin{tabular}{llll}
\hline Probe & Sequence & Target & Reference \\
\hline Plas18S & 5'-TTTAATAACTCGTTATATATATCAGTGTAGCAC-3' & Plasmodium spp. & Dinhopl et al. 2015 \\
Haemo18S_1 & 5'-GCTAACCGTAGTTATAGTCGCCATCTC-3' & Haemoproteus (Parahaemoproteus) spp. & Himmel et al. 2019 \\
\hline Leuc018S_1 & 5'-TAGGACTCCCCACTTGTCTTTTTCTTGA-3' & Leucocytozoon (Leucocytozoon) spp. & Himmel et al. 2019 \\
\hline Pmat18S_1b & 5'-CTAAAAGCATCTTGACTGTTACATCAAGACG-3' & Plasmodium matutinum (LINN1, AFTRU5) & present study \\
\hline Pvau18S_1b & 5'-CAAGCCGAAACTAATTACATTTAAACATAAGATCC-3' & Plasmodium vaughani (SYAT05) & present study \\
\hline
\end{tabular}

Table 2 Prevalence and diversity of haemosporidian parasites recorded in Eurasian blackbirds (Turdus merula) and song thrushes (T. philomelos) by PCR screening

\begin{tabular}{|c|c|c|c|c|c|}
\hline \multirow{2}{*}{$\begin{array}{l}\text { Host } \\
\text { species }\end{array}$} & \multirow{2}{*}{$\begin{array}{c}n \\
\text { examined }\end{array}$} & \multicolumn{3}{|c|}{ PCR positive ${ }^{a}$} & \multirow[t]{2}{*}{ parasite species \& cytb lineages ${ }^{b}$} \\
\hline & & Plas & HaemLeuc & total & \\
\hline $\begin{array}{l}\text { Turdus } \\
\text { merula }\end{array}$ & 277 & $166 / 32$ & $212 / 413 / 11$ & $179 / 35$ & $\begin{array}{l}\text { P. matutinum LINN1 (91/26), AFTRU5 (4/1); P. vaughani SYAT05 (88/26); P. elongatum GRW06 (1); } \\
\text { Plasmodium sp. TUMER12 (2), TUMER13 (1), TUMER14 (1/1), TUMER15 (1/1), TUMER16 (1); H. } \\
\text { minutus TURDUS2 (11/3), TUCHR01 (1/1), TUMER17 (1/1); Leucocytozoon sp. TUMER01 (7/7), } \\
\text { TUMER10 (1), ASOT06 (3/2), AEFUN03 (1/1), TUMER18 (1/1) }\end{array}$ \\
\hline $\begin{array}{l}\text { Turdus } \\
\text { philomelos }\end{array}$ & os & $11 / 2$ & $3 / 2 \quad 4 / 4$ & $15 / 5$ & $\begin{array}{l}\text { P. matutinum LINN1 (8/2); P. vaughani SYAT05 (1); P. circumflexum TURDUS1 (1); Plasmodium sp. } \\
\text { TUPHI08 (1/1), TUPHI09 (1); H. minutus TUPHI01 (3/2); Leucocytozoon sp. EUSE2 (2/2), TUPHI06 } \\
\text { (1/1), TUPHI10 (2/2), TUPHI11 (1/1), TUPHI12 (1/1) }\end{array}$ \\
\hline Total & 306 & $177 / 34$ & 4 15/6 17/15 & $194 / 40$ & 12 Plasmodium, 4 Haemoproteus, 10 Leucocytozoon lineages \\
\hline
\end{tabular}

${ }^{a}$ Numbers indicate total infected birds, followed by number of mixed infections

${ }^{b}$ Numbers in parentheses indicate total infected birds, followed by number of mixed infections. Lineages in bold are new cytB haplotypes

Table 3 Plasmodium exo-erythrocytic parasite load in Eurasian blackbirds (Turdus merula) and song thrushes (T. philomelos) determined by chromogenic in situ hybridization (CISH).

\begin{tabular}{|c|c|c|c|c|c|c|}
\hline \multirow[t]{2}{*}{ Host species } & \multirow{2}{*}{$\begin{array}{c}n \\
\text { examined }\end{array}$} & \multicolumn{5}{|c|}{ exo-erythrocytic stages detected by CISH } \\
\hline & & negative & low ${ }^{a}$ & moderate $^{a}$ & high $^{a}$ & total positive \\
\hline Turdus merula & $166(32)$ & $17(3)$ & $66(9)$ & $33(4)$ & $50(16)$ & $149(29)$ \\
\hline Turdus philomelos & $11(2)$ & $1(0)$ & $8(1)$ & $1(0)$ & $1(1)$ & $10(2)$ \\
\hline Total & $177(34)$ & $18(3)$ & $74(10)$ & $34(4)$ & $51(17)$ & $159(31)$ \\
\hline
\end{tabular}

Numbers in parentheses indicate mixed infections with either multiple Plasmodium lineages or Haemoproteus and Leucocytozoon lineages 
${ }^{a}$ Numbers of cases exhibiting low, moderate, and high exo-erythrocytic parasite loads in at least one of the examined organs

\section{Additional Files}

Additional file 1 Plasmodium parasite stages visualized by the dark-purple $\mathrm{CISH}$-signal in a histological section of the liver. Erythrocytic and exo-erythrocytic stages were distinguished by the size, shape and location of the signals: blood stages showed roundish to oval signals usually not larger than blood cells and were located in capillaries and larger vessels (arrowheads), whereas signals of tissue stages usually exceeded the size of blood cells and showed variable shapes (arrows). Scale bar. $20 \mu \mathrm{m}$

Additional file 2 Representative photographs showing low- (left), moderate- (middle) and high-grade (right) exo-erythrocytic parasite load in histological sections of the brain (a-c) and the spleen (d-f) determined by chromogenic in situ hybridization. Scale bar. $100 \mu \mathrm{m}$

\section{Figures}

\section{Figure 1}

Location of exo-erythrocytic stages of Plasmodium spp. in Eurasian blackbirds and song thrushes $(\mathrm{n}=$ 177). The spleen was most commonly parasitized with an infection rate of $86.7 \%$ (144/166), followed by the lung with $65.0 \%(93 / 143)$ and the brain with $61.0 \%(100 / 164)$.

\section{Figure 2}

Histological sections of the intestine (a), brain (b), heart (c) and gizzard (d) from infected Eurasian blackbirds (Turdus merula) showing developmental stages of Plasmodium matutinum and P. vaughani. Parasites were visualized by $\mathrm{CISH}$ (a-d, inserts, low magnification) and identified in haematoxylin-eosin stained tissue sections (a-d, high magnification). (a) In the intestine, numerous meronts (arrowheads) were located in cytoplasm of large cells (presumably macrophages) of the lamina propria mucosae accompanied by severe displacement and atrophy of crypt structures. (b) In the brain, clusters of meronts were occasionally observed in several birds. Some of the meronts showed multiple merozoites-containing compartments (white arrowheads). (c, d) Erythrocytic parasite stages (arrows) were frequently observed to accumulate in fat tissue (c) and in the subserosal layers (d) of visceral organs. Scale bars: $25 \mu \mathrm{m}$, inserts: $50 \mu \mathrm{m}$

\section{Figure 3}


Comparison of exo-erythrocytic parasite load in different organs of Eurasian blackbirds (Turdus merula) infected with Plasmodium matutinum LINN1 $(n=65)$ and P. vaughani SYAT05 $(n=62)$. Birds infected with P. matutinum LINN1 showed more frequently moderate- and high-grade parasite load in almost all organs compared to birds infected with P. vaughani SYAT05 (Fisher's exact: heart, lung, liver, spleen, kidney, brain, gizzard, intestine), ${ }^{*} p<0.05,{ }^{* *} p<0.001$ ).

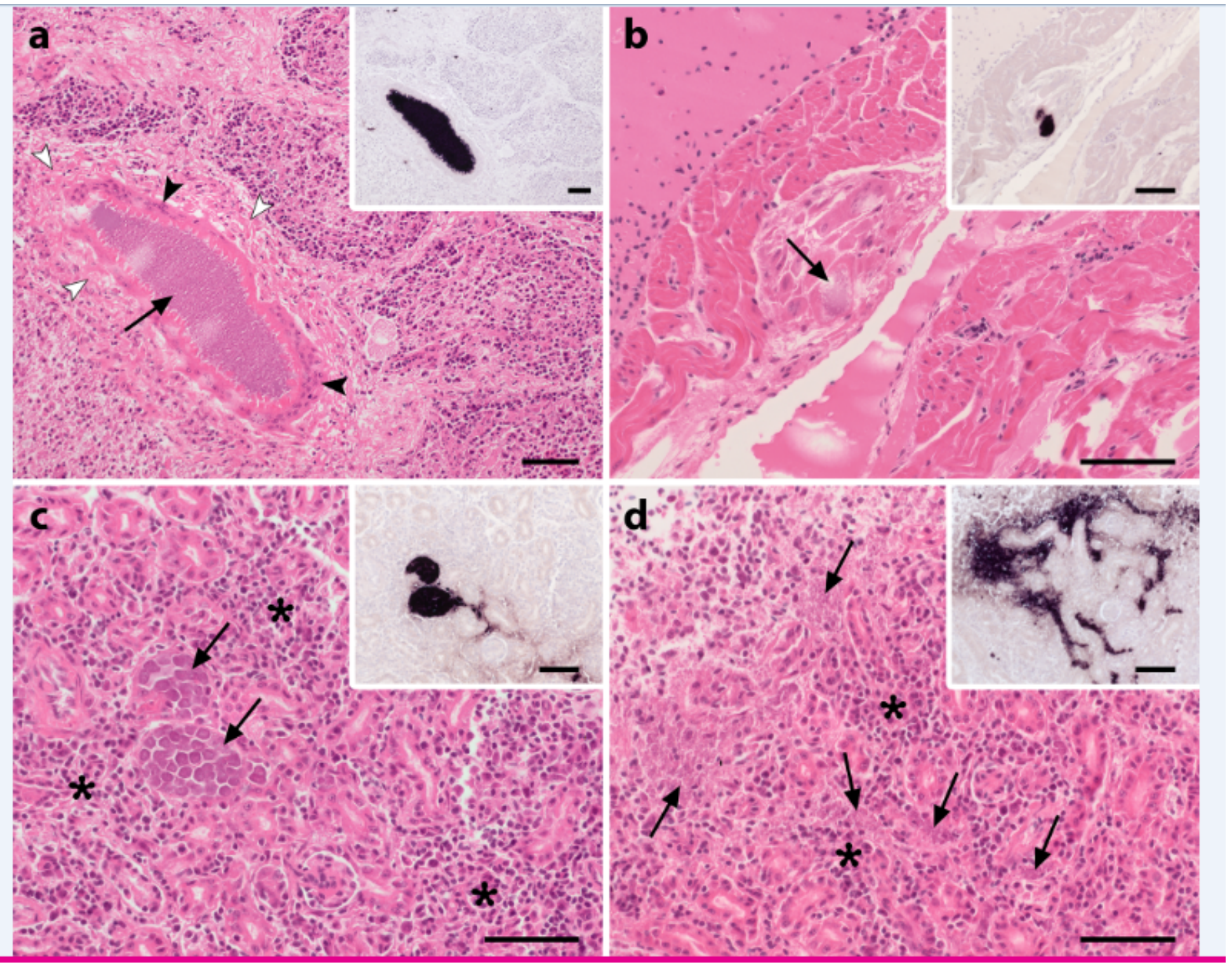

\section{Figure 4}

Tissue stages of Leuocytozoon sp. TUMER01 in histological sections of two Eurasian blackbirds (Turdus merula). Megalomeronts (arrows) were detected by CISH (inserts) and identified in HE-stained sections of the bursa of Fabricius (a), heart (b) and kidney (c, d). Megalomeronts consisted of densely packed homogenous masses of merozoites $(a, b)$, surrounded by cells of unclear origin (arrowheads) or featured cytomeres enclosed by a thin wall (c). In the kidney, numerous merozoites of 1-2 $\mu \mathrm{m}$, barely identifiable in HE-stained sections, were located in the interstitial tissue (arrows), resulting in a strong, scattered CISH signal between renal tubules (d). Histopathological changes such as fibrosis (a, white arrowheads) and 
non-suppurative inflammation (c, d, asterisks) were associated with the parasite stages. Scale-bars: 50 $\mu \mathrm{m}$

\section{Supplementary Files}

This is a list of supplementary files associated with this preprint. Click to download.

- Additionalfile1.tif

- Additionalfile2.tif 